\title{
Medical Cannabis Use Among Older Adults in Canada: Self-Reported Data on Types and Amount Used, and Perceived Effects
}

\author{
Shankar Tumati ${ }^{1} \cdot$ Krista L. Lanctôt $^{1,2,3}$ (D) RuoDing Wang ${ }^{1} \cdot$ Abby Li $^{1} \cdot$ Andrew Davis ${ }^{4} \cdot$ Nathan Herrmann ${ }^{1,2}$
}

Accepted: 2 December 2021 / Published online: 23 December 2021

(c) The Author(s), under exclusive licence to Springer Nature Switzerland AG 2021

\begin{abstract}
Background Medical cannabis use is growing among older adults. In this retrospective study, we aimed to assess the characteristics of older medical cannabis users including the indications, type and amount of cannabis used, perceived changes in symptoms after cannabis use, change in dose of concurrent medications, and adverse effects.

Methods Data were collected between October 2014 and October 2020 from patients who were consulting the Canada-wide network of clinics of a medical cannabis provider and who were willing to answer questionnaires based on their medical status. The current study included older adults ( $\geq 65$ years) who completed questionnaires at intake and first follow-up visits. Data were summarized with descriptive statistics, which were compared between men and women with $t$ tests or chi-squared tests. Tests of proportions assessed categorical responses for perceived effects after cannabis use. Logistic regression was used to assess trends in cannabis usage.

Results Data included that from 9766 older adult users at intake ( $m e a n+\mathrm{SD}$ age $=73.2 \pm 6.8$ years, females $=60.0 \%$ ), among whom 4673 (females $=61.4 \%$ ) returned for follow-up after $90.6 \pm 58$ days. The most common primary indication for which medical cannabis was sought was pain $(67.7 \%)$, which was more common in women, whereas oncological and neurological conditions were more common in men. At follow-up, cannabis oil was used by $81.0 \%$ of older adults, among whom compositions containing only or mostly cannabidiol (CBD) had been used by $83.6 \%$. Adverse effects reported by older adults at the follow-up visit included dry mouth (12.8\%), drowsiness (8.6\%), and dizziness (4.0\%). The majority of older adults reported improvements in pain $(72.7 \%, z=1482.6, p<0.0001$, compared to worsening or no change), sleep (64.5\%, $z=549.4, p<0.0001)$, and $\operatorname{mood}(52.8 \%, z=16.4, p<0.0001)$, with $35.6 \%$ reporting use of a reduced dose of opioids and $19.9 \%$ a reduced dose of benzodiazepines.

Interpretation Among older adults, medical cannabis is used more often by women, with CBD-containing cannabis oils being the most commonly used. Users reported improved pain, sleep, and mood symptoms at follow-up after cannabis use. This study describes the patterns of use of medical cannabis by older adults and highlights the need for research to determine appropriate indications, precise doses of active ingredients, and short- and long-term outcomes among older adults.
\end{abstract}

Krista L. Lanctôt

krista.lanctot@sunnybrook.ca

1 Neuropsychopharmacology Research Group, Sunnybrook Research Institute, Toronto, ON, Canada

2 Department of Psychiatry, University of Toronto, Toronto, ON, Canada

3 Department of Pharmacology and Toxicology, University of Toronto, Toronto, ON, Canada

4 Department of Economics, Acadia University, Wolfville, NS, Canada

\section{Key Points}

Older adults used medical cannabis most often for pain, and use was more common among women.

The most commonly used form was cannabis oil; most users consumed $<2 \mathrm{ml} /$ day and favoured compositions containing mostly or almost exclusively cannabidiol.

A majority of older adults reported improvement in pain, sleep, and mood after medical cannabis use. 


\section{Introduction}

Cannabis has been used globally for medical and nonmedical purposes [1]. In the 20th century, several countries prohibited cannabis use due to concerns over its abuse potential [2]. However, attitudes towards cannabis have shifted over the last 3 decades possibly due to the perceived benefits for health problems, the challenges faced by the legal system in reducing use, and the costs of implementing prohibitory laws [3]. Canada has been at the forefront of these developments [4] and was among the first countries in the world to develop a legal and regulatory framework for therapeutic cannabis use by severely ill patients [5]. Under that framework, medical cannabis was accessed by over 300,000 patients in 2020 [6]. Moreover, in 2012, despite being illegal at that time, $42.5 \%$ of Canadians reported using cannabis at least once over their lifetime [7]. The high rates of cannabis use combined with a shift in public opinion led to the legalization of nonmedical cannabis use in 2018 in Canada $[8,9]$.

National Canadian surveys estimate that the percentage of those using cannabis in the past year grew from $9.4 \%$ in 2004 to $14.0 \%$ in 2014 , and further from $17.5 \%$ in 2019 (past 3 months use, post-legalization) to $20.0 \%$ (past 3 months use) in 2020 [10]. Over that period, adults aged 65 years and above were the fastest growing user age group [11-13], increasing from $0.8 \%$ in 2012 [7] to $6.6 \%$ in 2019 , including $27.0 \%$ first-time (past 3 months) users [13]. A similar increase in older cannabis users has been reported in other countries [14-16]. While men use cannabis more often, rates of cannabis use among women have increased in recent years $[10,17]$. Despite the increasing number of publications on medical cannabis [18], there is a dearth of information about its use among older adults. For example, surveys by Health Canada provide aggregate data for all adults aged 25 years and above [5, 17]. Consequently, older adults who are more likely to source cannabis legally, rely on non-clinical sources to guide cannabis use [19-21]. At the same time, physicians report concerns about cannabis use among older adults due to a lack of evidence for benefits and harms [22]. As such, Canadian clinical practice guidelines recommend restricted use of synthetic cannabinoids (e.g., nabilone and nabiximols) only for neuropathic pain, palliative care, nausea and vomiting associated with chemotherapy, and spasticity due to multiple sclerosis or spinal cord injury, while recommending against cannabinoids for other conditions [23]. Notably, no age-specific recommendations are provided.

Older adults use cannabis for medical conditions more often than young and middle-aged adults [24, 25], with more than half $(52.0 \%)$ reporting medical use exclusively, and an additional $23.6 \%$ reporting cannabis use for medical and non-medical purposes [13]. These conditions include chronic pain due to musculoskeletal disorders and cancer, chemotherapy-associated nausea, vomiting and loss of appetite, and various neurological and inflammatory conditions [26, 27]. Cannabis has also been used for mental health conditions such as anxiety, depressive symptoms, insomnia, and post-traumatic stress disorder [14, 27]. Among older adults, adverse events, which include dizziness, dry mouth, somnolence, and falls, are of concern $[28,29]$ and may be more related to tetrahydrocannabinol (THC) than cannabidiol (CBD) [28]. Other concerns surrounding cannabis use among older adults include the presence of one or more medical ailments, use of multiple medications, and altered metabolic processing of drugs [30].

Few studies have reported on the characteristics and patterns of medical cannabis use among older adults, including differences between males and females [31, 32]. In this study, we describe the characteristics, the type and amount of medical cannabis used, and self-rated effectiveness, adverse effects, and change in concomitant medications. We also assessed sex differences in these variables.

\section{Methods}

\subsection{Study data}

Anonymized data included in this study were collected between October 2014 and October 2020 by a commercial medical cannabis provider (Canabo Medical Clinic) based in Canada. These data were routinely collected for use by physicians of the clinic. Users were either referred to the clinic by physicians for symptom management or were selfreferred. All users aged 65 years and above with fully or partially completed questionnaires were included in this study. Completion of the questionnaire was voluntary but encouraged, and users were asked for consent to use their data for research purposes. Data analyses were approved by the Sunnybrook Research Ethics Board.

Intake visit As part of the initial consult, users consulted a physician and received a prescription. If needed, a trained medical cannabis educator then provided users with information on the products available (cannabinoid oil, herbal cannabis, and synthetic cannabinoids) and route of intake, in compliance with the prescription. During this visit, users answered questions about their demographic, social, and health details (see the electronic supplementary material, Supplementary Information [SI]_-SI Methods').

Follow-up visit At follow-up visits, which occurred over variable durations, users were asked to complete another questionnaire about cannabis products used, symptoms, adverse effects, and changes in use of concurrent 
medications. These questions determined the type and amount of cannabis used daily, and their content of THC and CBD. For the present study, we focused on cannabis oils and herbal cannabis as these products were predominantly used by patients across the duration of data collection (89.4\%). Other products such as Sativex ${ }^{\circledR}$ spray were excluded from the questionnaire in 2020 due to low use and replaced by capsules and new spray forms. Patients were asked to rate their symptoms of pain, mood, and sleep since starting cannabis use under the following categories: ' $>75 \%$ better', ' $50-75 \%$ better', ' $25-50 \%$ better', ' $0-25 \%$ better', and 'none/worse'. Symptoms were not queried at the intake visit. Patients were also asked if they experienced adverse effects, which were listed for selection and included dry mouth, drowsiness, dizziness, anxiety, and hallucinations. Users were also asked if the dose of concomitant medications used had changed since starting medical cannabis, with the following options for selection: 'significantly decreased', 'mildly decreased', 'no change', 'mildly increased', 'significantly increased', and 'unsure'. These data are reported as number and percentage of users in each category at the follow-up visit.

Sample sizes for each measure vary due to incomplete questionnaires, questionnaire changes over time, and patient attrition. In addition, users were able to exit the questionnaire before completion but were not allowed to skip questions; hence, data available for individual questionnaire items differed. The resulting sample size for each item is provided in parentheses where required.

\subsection{Statistical analyses}

Demographic information was summarized using descriptive statistics and expressed as mean (standard deviation [ $\pm \mathrm{SD}]$ ) for continuous variables and in percentages for categorical variables. Sex differences were assessed for demographic variables at intake and follow-up visits with $t$ tests and chi-squared tests for continuous and categorical data, respectively. Sex differences in composition of medical cannabis used, ratings of symptom changes, individual adverse effects, and changes in medication dose were assessed with tests of proportions. In addition, user-rated changes in symptoms after cannabis use were converted into a ratio of those reporting improvement ( $0-25 \%$ better, $25-50 \%$ better, $50-75 \%$ better, $>75 \%$ better) to those reporting no change or worsening of symptoms. For change in doses of concomitant medications, we calculated a ratio of the number of patients reporting use of a reduced dose to those reporting use of an increased dose. The two derived ratios for symptom rating and change in medication doses were each compared with chi-squared tests. Trends over time for the proportion of older adults attending the clinics $(<65$ years or $\geq 65$ years), proportion of older women (compared to older men) attending the clinics, and type of cannabis (cannabis oil or herbal cannabis) used were assessed with logistic regression with time as the predictor variable. Data analyses were performed using STATA v15.1 (College Station, Texas). All statistical tests were considered significant at a two-sided $p$ value $<0.05$.

\section{Results}

\subsection{Characteristics of Older Adult Users of Medical Cannabis}

The current study included data from 9766 older adults (65 years and above, $60.0 \%$ female) who completed the intake questionnaire (Fig. 1). Among them, 4673 users (61.4\% female) completed at least one follow-up visit. The interval between the intake and the follow-up visit was 90.6 $(\mathrm{SD} \pm 58)$ days. The demographic characteristics of older adults at the intake visit are shown in Table 1. Data for patients who also attended a follow-up visit are shown in SI Table 1 (see the Electronic Supplementary Material). At intake, older adult users had a mean age of $73.2(\mathrm{SD} \pm 6.8)$ years (median $=72.0$ years) and women were older than men. More women than men attended the clinic (intake visit: $z=19.7, p<0.0001$; follow-up visit: $z=15.6, p$ $<0.0001$ ). These patients represented $23.1 \%$ of all users attending the medical cannabis clinic who completed the intake questionnaire $(n=42,267$, mean age $=51.5 \pm 16.8$ years). Older adult users resided in Ontario (57.9\%), Alberta (16.3\%), Newfoundland and Labrador (15.0\%), or Nova Scotia $(9.1 \%)(n=7440)$. Among them, $64.4 \%$ were married or in common-law relationships, $28.2 \%$ were divorced, and $7.4 \%$ were single $(n=9334)$. Previous cannabis use was reported by $15.5 \%$ of older adults, which was more common in men than women. Older adults were most frequently referred to the clinic for pain disorders, and the most common concomitant medications were overthe-counter analgesics and opioids (Table 1). SI Table 2 shows that arthritis, chronic pain, and lower back pain were the most frequent pain conditions. Pain was more common in women than men, whereas oncological and neurological conditions were more common in men than women. All concomitant medications except opioids were used more often by women than men.

\subsection{Type and Amount of Medical Cannabis Use Among Older Adults}

At follow-up, $81.0 \%$ of older adults reported using cannabis oils and $11.1 \%$ reported using herbal cannabis $(n=4302)$. More men than women used herbal cannabis 


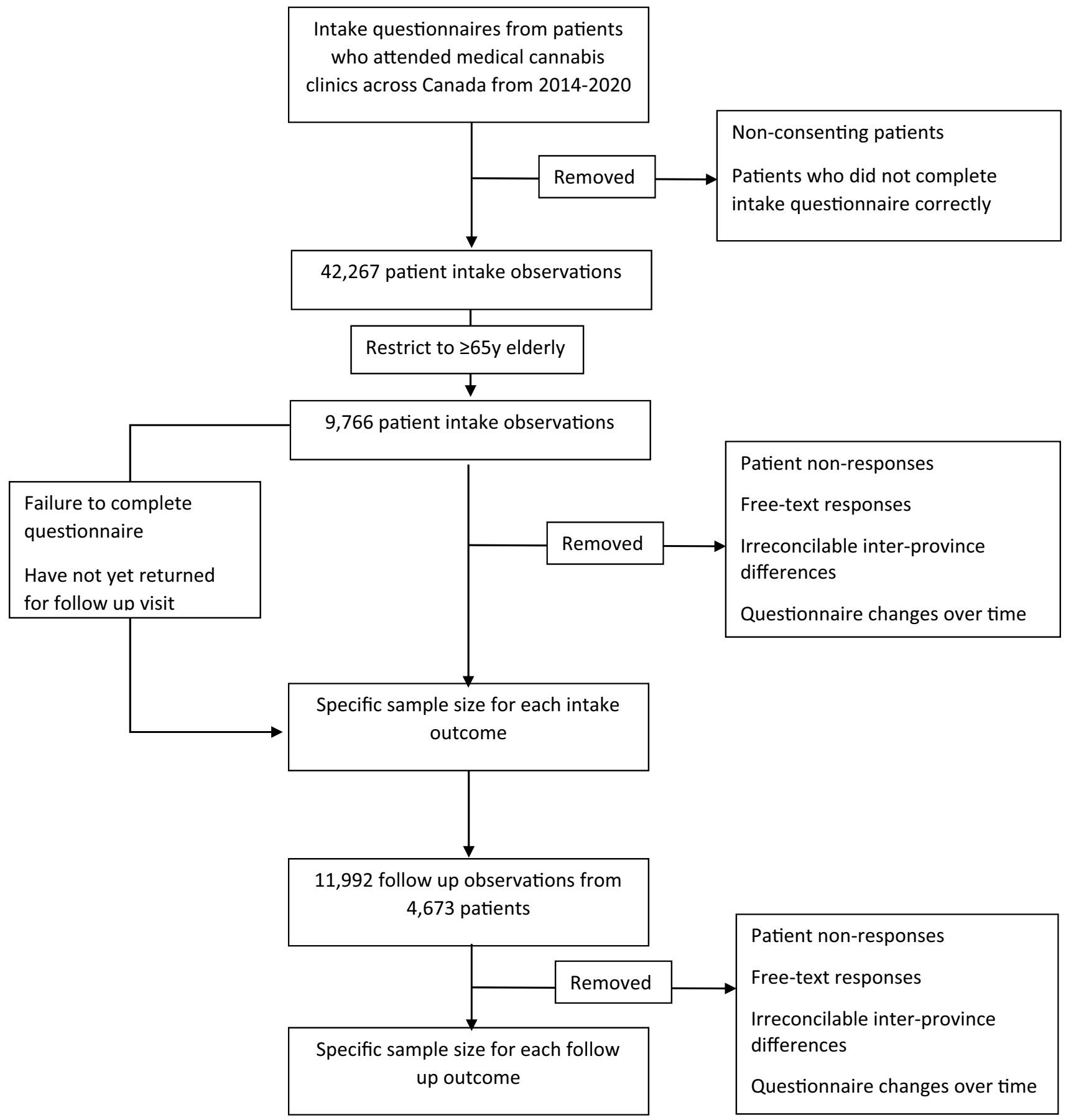

Fig. 1 Data inclusion flowchart

$(z=6.44 ; p<0.0001)$ whereas more women than men preferred cannabis oils $(z=3.87 ; p=0.0001)$.

Among those taking cannabis oils at follow-up $(n=2495)$, the majority consumed less than $2 \mathrm{~mL} /$ day (Table 2). Older adults preferred to use CBD over THC (almost exclusively or mostly CBD [83.6\%] vs almost exclusively or mostly THC [4.4\%]). Over half of older adults used almost exclusively CBD oils (males $=50 \%$, females $=53.2 \%$ ), whereas only $2.3 \%$ of men and $1.6 \%$ of women among older adults used oils containing THC almost exclusively. Men and women did not differ in amounts $\left(\chi^{2}=1.19, p=0.88\right)$ or composition $\left(\chi^{2}=7.74\right.$, $p=0.10$ ) of cannabis oils used. The amount of herbal cannabis used was less than $1.5 \mathrm{~g} /$ day for the majority of older adults (SI Table 3; see the electronic supplementary 
Table 1 Demographic characteristics of older medical cannabis users at the intake visit

\begin{tabular}{|c|c|c|c|c|}
\hline & $\begin{array}{l}\text { Older adults } \\
(n=9766)\end{array}$ & $\begin{array}{l}\text { Men } \\
(n=3908)\end{array}$ & $\begin{array}{l}\text { Women } \\
(n=5856)^{*}\end{array}$ & Men vs women** \\
\hline Age $($ mean $\pm S D)$ & $73.2 \pm 6.8$ & $72.5 \pm 6.2$ & $73.6 \pm 7.1$ & $\begin{array}{l}t=7.93 \\
p<0.0001\end{array}$ \\
\hline$\%$ Prior cannabis use & $15.5 \%(n=7230)$ & $19.5 \%(n=2794)$ & $13.0 \%(n=4434)$ & $\begin{array}{l}z=7.44 \\
p<0.0001\end{array}$ \\
\hline$\%$ Smoking & $11.3 \%(n=7441)$ & $11.6 \%(n=2794)$ & $11.1 \%(n=4566)$ & $\begin{array}{l}z=-0.65 \\
p=0.52\end{array}$ \\
\hline$\%$ Alcohol use & $62.2 \%(n=9765)$ & $66.1 \%(n=3908)$ & $59.6 \%(n=5855)$ & $\begin{array}{l}z=-6.44 \\
p<0.0001\end{array}$ \\
\hline Medications & $n=9620$ & $n=3842$ & $n=5776$ & \\
\hline OTC analgesics & $44.5 \%$ & $37.3 \%$ & $49.3 \%$ & $\begin{array}{l}z=11.62 \\
p<0.0001\end{array}$ \\
\hline Opioids & $28.3 \%$ & $27.9 \%$ & $28.6 \%$ & $\begin{array}{l}z=-0.66 \\
p=0.51\end{array}$ \\
\hline NSAIDs & $24.5 \%$ & $23.3 \%$ & $25.3 \%$ & $\begin{array}{l}z=2.25 \\
p=0.024\end{array}$ \\
\hline Antidepressants & $21.4 \%$ & $16.3 \%$ & $24.8 \%$ & $\begin{array}{l}z=9.97 \\
p<0.0001\end{array}$ \\
\hline Nerve modulators & $14.3 \%$ & $12.9 \%$ & $15.3 \%$ & $\begin{array}{l}z=3.38 \\
p=0.0007\end{array}$ \\
\hline Benzodiazepines & $12.3 \%$ & $9.1 \%$ & $14.4 \%$ & $\begin{array}{l}z=7.81 \\
p<0.0001\end{array}$ \\
\hline Primary diagnosis & $n=9620$ & $n=3842$ & $n=5776$ & \\
\hline Pain & $67.7 \%$ & $63.3 \%$ & $70.6 \%$ & $\begin{array}{l}z=7.52 \\
p<0.0001\end{array}$ \\
\hline Oncological & $10.1 \%$ & $12.7 \%$ & $8.4 \%$ & $\begin{array}{l}z=-6.88 \\
p<0.0001\end{array}$ \\
\hline Psychiatric & $7.9 \%$ & $8.4 \%$ & $7.6 \%$ & $\begin{array}{l}z=-1.33 \\
p=0.18\end{array}$ \\
\hline Neurological & $7.0 \%$ & $8.8 \%$ & $5.8 \%$ & $\begin{array}{l}z=-5.60 \\
p<0.0001\end{array}$ \\
\hline Others & $7.3 \%$ & $6.8 \%$ & $7.6 \%$ & $\begin{array}{l}z=1.31 \\
p=0.19\end{array}$ \\
\hline
\end{tabular}

Nerve modulators include pregabalin and gabapentin for pain; antiepileptics include carbamazepine, valproic acid, phenytoin, and lamotrigine. A breakdown of primary diagnosis indications in the categories of pain, psychiatric disorders, and neurological disorders is shown in the Electronic Supplementary Material (SI Table 2)

NSAID nonsteroidal anti-inflammatory drug, OTC over the counter, SI supplementary information

*Two users identified as "other" gender (introduced in the questionnaire in 2019) and were excluded from summary statistics and statistical comparisons

**Statistics reported are $t$ or $z$ statistic and $p$ value

material), and was higher in men than women $\left(\chi^{2}=9.64\right.$, $p=0.047)$.

\subsection{Self-Reported Adverse Effects After Beginning Medical Cannabis Use}

At follow-up, among older adults using cannabis oils $(n=$ 3009 ), at least one adverse effect was reported by $23.7 \%$ of men $(n=1097)$ and $26.6 \%$ of women $(n=1912)$. These rates did not differ between men and women $(z=3.08 ; p=0.08)$. The most common adverse effects were dry mouth (12.8\%), drowsiness (8.6\%), and dizziness (4.0\%). Anxiety was reported by $1.3 \%$ and hallucinations by $0.5 \%$. 
Table 2 Daily amount and composition of cannabis oils used by older adults (reported as number and percentage of all older adult users of cannabis oils)

\begin{tabular}{llccrcr}
\hline mL/day & Across composition & Almost exclusively CBD & $\begin{array}{l}\text { Mostly CBD, } \\
\text { some THC }\end{array}$ & $\begin{array}{l}\text { Equal parts CBD } \\
\text { and THC }\end{array}$ & $\begin{array}{l}\text { Mostly THC, } \\
\text { some CBD } \\
\text { Almost } \\
\text { exclusively } \\
\text { THC }\end{array}$ \\
\hline & & $1298(52.0 \%)$ & $788(31.6 \%)$ & $299(12.0 \%)$ & $64(2.6 \%)$ & $46(1.8 \%)$ \\
$0-0.5$ & $369(14.8 \%)$ & $181(7.3 \%)$ & $99(4.9 \%)$ & $57(2.3 \%)$ & $16(0.6 \%)$ & $16(0.6 \%)$ \\
$0.5-1$ & $752(30.1 \%)$ & $362(14.5 \%)$ & $246(9.9 \%)$ & $108(4.3 \%)$ & $22(0.8 \%)$ & $14(0.6 \%)$ \\
$1-1.5$ & $487(19.5 \%)$ & $258(10.3 \%)$ & $158(6.3 \%)$ & $56(2.2 \%)$ & $10(0.4 \%)$ & $5(0.2 \%)$ \\
$1.5-2$ & $356(14.3 \%)$ & $201(8.0 \%)$ & $119(4.8 \%)$ & $29(1.2 \%)$ & $5(0.2 \%)$ & $2(<0.1 \%)$ \\
$>2$ & $531(21.3 \%)$ & $296(11.9 \%)$ & $166(6.7 \%)$ & $49(2.0 \%)$ & $11(0.4 \%)$ & $9(0.4 \%)$ \\
\hline
\end{tabular}

Older adults prefer CBD over THC composition. The table also shows the daily dose of cannabis oil according to composition used by the number and percentage of older adults. Although the precise dose of cannabis consumed by users was not available, most cannabis oil products in the marketplace containing almost exclusively CBD are formulated at $20 \mathrm{mg} / \mathrm{mL}$. In the case of CBD and THC containing oils, the concentrations of active ingredients in available products vary, ranging from 20 to $50 \mathrm{mg} / \mathrm{mL} \mathrm{CBD}$ in combination with $<1 \mathrm{mg} / \mathrm{mL}$ to $2 \mathrm{mg} / \mathrm{mL}$ THC

CBD cannabidiol, $T H C$ tetrahydrocannabinol

\subsection{Self-Reported Perception of Symptoms and Medication Use After Beginning Medical Cannabis Use}

At follow-up, users were asked to rate if there were changes in their symptoms of pain, mood, and sleep since starting medical cannabis $(n=4303)$ (Fig. 2). A larger proportion of users reported improvement versus no change or worsening in these symptoms (pain symptoms [ $n=3597]$, ratio 2.6, $z=1482.6, p<0.0001$; sleep [ $n=3267]$, ratio $1.8, z=$ 549.4, $p<0.0001 ; \operatorname{mood}[n=2623]$, ratio $1.1, z=16.4, p$ $<0.0001)$. No differences were found in the proportions of men and women rating pain $\left(\chi^{2}=2.18, p=0.70\right)$, sleep $\left(\chi^{2}\right.$ $=8.65, p=0.12)$, or $\operatorname{mood}\left(\chi^{2}=3.51, p=0.62\right)$ symptoms.

Older adults were also asked if their doses of concomitant medications changed after starting medical cannabis use. Adequate data were available for four classes of drugs: opioids, nerve modulators, antidepressants, and benzodiazepines (Fig. 3). Across those drug classes, the majority of older adults reported that the dose remained unchanged, while $35.6 \%$ and $19.9 \%$ of older adults reported using a reduced dose of opioids and benzodiazepines, respectively. However, the proportion of those reporting use of a reduced dose was larger than those reporting use of an increased dose for all drug categories (opioids: ratio 4.5, $z=179.9, p<0.0001$; nerve modulators: ratio $2.1, z=16.9, p<0.0001$; antidepressants: ratio 2.8, $z=25.9, p<0.0001$; benzodiazepines: ratio 5.2, $z=$ $41.3, p<0.0001)$. The percentages of men and women in each category of change in medication doses were similar (nerve modulators: $\chi^{2}=4.48, p=0.48$; antidepressants: $\chi^{2}=3.75, p=0.59$; benzodiazepines: $\chi^{2}=5.32, p$ $=0.38$ ) except for opioids (opioids: $\chi^{2}=17.83, p=0.003$, more men reported using 'significantly decreased' doses of opioids $[z=-2.35, p=0.018$, compared to the rest of the categories]).

\subsection{Time Trends in Medical Cannabis Use Among Older Adults}

Between 2014 and 2020, the proportion of older adults increased from $17.6 \%$ (total sample across all ages, $n=$ $18555)$ pre-2018 to $31.2 \%(n=8869)$ in 2019 (odds ratio $[\mathrm{OR}]=1.20, p<0.001)$, although this number decreased to $22.7 \%$ in 2020 ( $n=5644)$, possibly due to the coronavirus disease 2019 (COVID-19) pandemic. Furthermore, the proportion of women among older adults increased from $56.5 \%$ pre- 2018 to $61.7 \%$ in $2020(\mathrm{OR}=1.08, p$ $<0.0001)$.

The types of cannabis used also changed over time. While herbal cannabis use declined from pre-2018 to 2020 (OR = $0.24 ; p<0.0001)$, use of cannabis oils among older adults increased $(\mathrm{OR}=1.66 ; p<0.0001)$ (Fig. 4).

\section{Discussion}

This study described medical cannabis use in a large cohort of older adults visiting a Canadian medical cannabis provider. At intake, $23.1 \%$ of all patients were older adults, and $60.0 \%$ of older adults were women. A larger proportion of older men $(19.5 \%)$ were prior cannabis users compared to women $(13.0 \%)$. The most common indication for medical cannabis use was pain, which was more common in women than men. Cannabis oil was used by $81.0 \%$ of older adults, among whom $78.7 \%$ used $<2 \mathrm{~mL} /$ day and $83.6 \%$ used almost exclusively or mostly CBD-containing cannabis oil. After cannabis use, among older adults who were treating 
Fig. 2 Percentage of older adults in each category of change in symptom severity at the follow-up visit after medical cannabis use (Sleep: $n=3267$, Pain: $n=3597$, Mood: $n=2623$ )
๑Sleep $\square$ Pain Mood

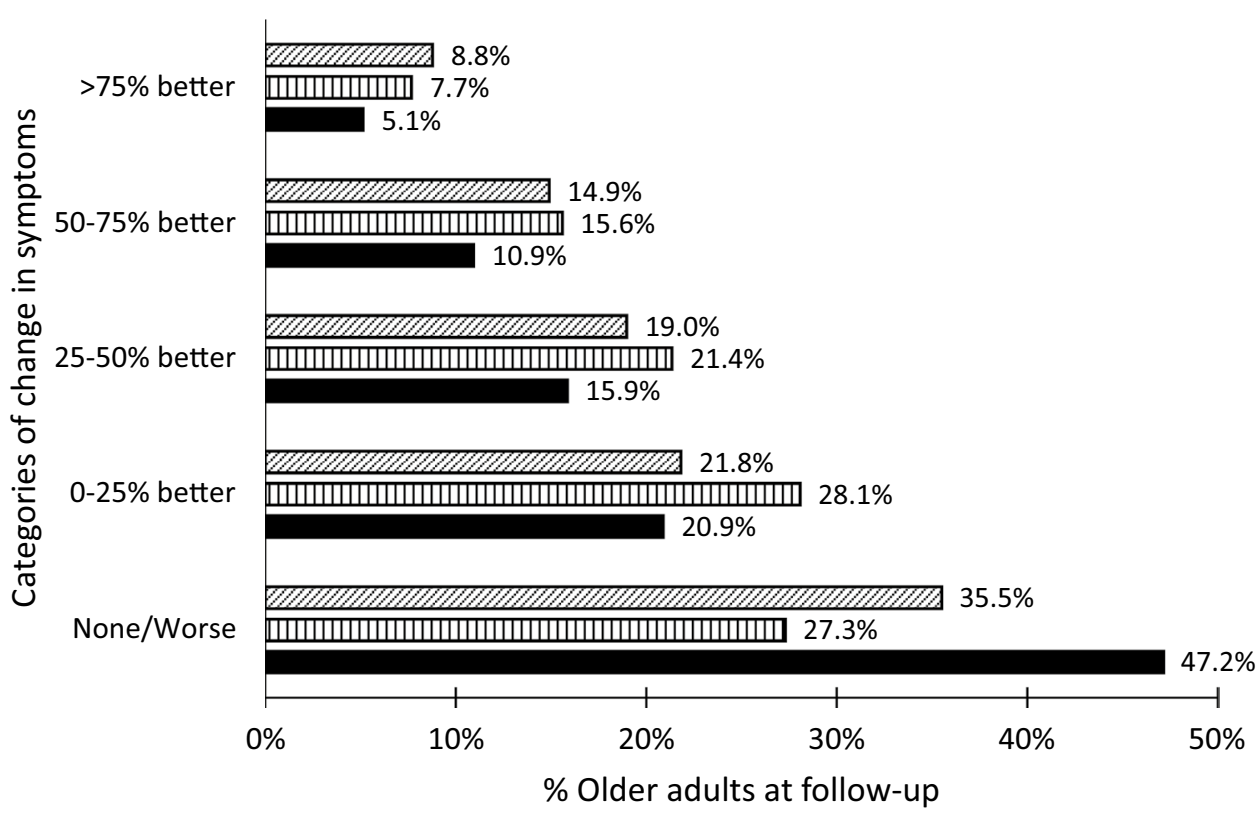

$\square$ Benzodiazepines $(n=341) \square$ Nerve modulators $(n=594)$

$\square$ Antidepressants $(n=939) \quad \square$ Opioids $(n=798)$

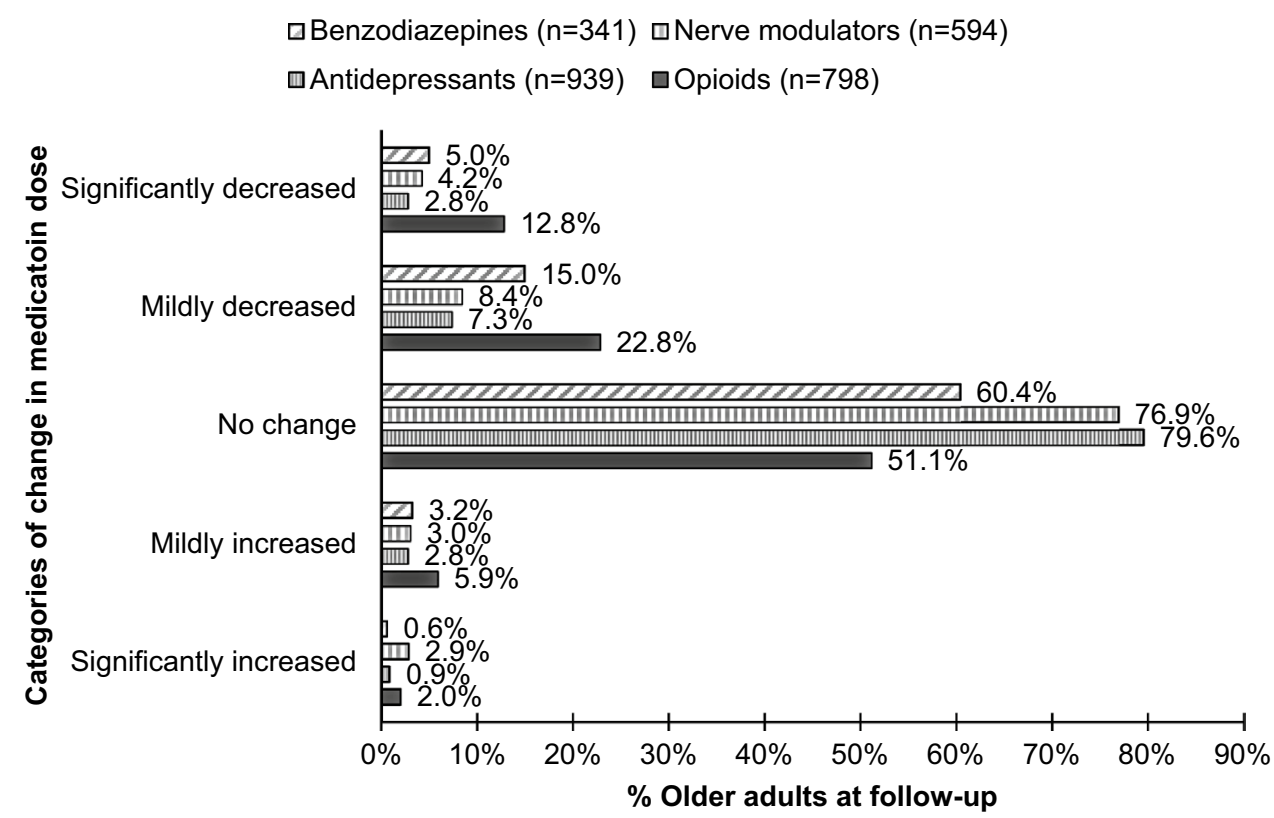

Fig. 3 Percentage of older adults in each category of change in dose of concomitant medications at the follow-up visit after starting medical cannabis use. Note that percentage of users who indicated 'unsure' about change in medication dose are not displayed (opioids $=5.4 \%$; nerve modulators $=4.5 \%$; antidepressants $=6.7 \%$; benzodiazepines $=15.8 \%$ ) specified symptoms, $72.7 \%$ rated having reduced pain, $64.5 \%$ rated having better sleep, and $52.8 \%$ reported better mood. Doses of concomitant medications were reported to be unchanged by a majority of older adults although $35.6 \%$ and $19.9 \%$ reported reduced doses of opioids and benzodiazepines, respectively. Over the study duration, the proportion of older adults attending the clinic increased, with the proportion of women increasing over time. Use of CBDcontaining cannabis oils increased over the study duration.
Together, these results show the characteristics of medical cannabis use in older adults including the amount and type of cannabis preferred.

Older adults in our sample comprised $31.2 \%$ of the clinics' users in 2019; in contrast, older adults comprised $7.6 \%$ of cannabis users in the quarterly internet-based National Cannabis Survey of Health Canada [13]. In a 2019 national survey conducted in the USA, adults over 50+ years comprised $9.6 \%$ of adult cannabis users [33]. Similar to our 


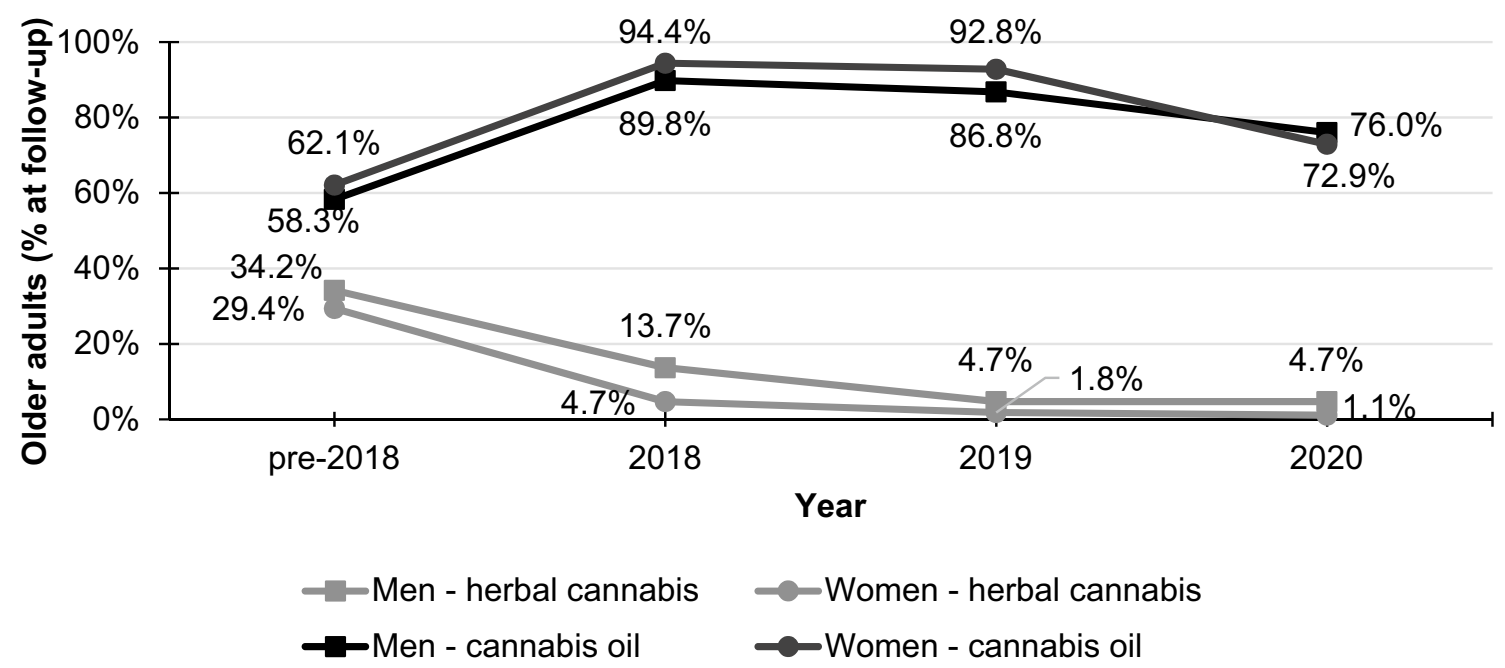

Fig. 4 Type of medical cannabis reported as being used by percentage of older adults by year (males $=1639$, females $=2663$ ). The percentages do not add up to $100 \%$ as a small minority of older adults used other forms of cannabis products or used multiple forms

study, among users $(n=7548)$ registered in a medical cannabis program, $23.6 \%$ were $\geq 65$ years old [27]. These results support reports that older adults are more likely to seek clinical sources for medical cannabis use than young and middle-aged groups [25]. Similar to other studies [19, 20, 26, 34-36], older adults in this study, particularly women, sought medical cannabis most often for pain relief. However, poor sleep and anxiety were less common than in community (38\% and 24\%) and clinic-based (29\% and 24\%) studies $[35,36]$. These differences may be due to a higher proportion of older adults seeking cannabis for medical purposes [25], biases in convenience sampling, though increasing social acceptance, easy accessibility, and high rates of selfmedication for mood and anxiety symptoms [37] may also be potential factors. In our results and those of others, older women were frequent medical cannabis users [35, 36, 38]. Although the proportion varies [19, 20, 39], women comprise approximately $60-65 \%$ of older adult users. Furthermore, previous research indicates that more older women are new users of cannabis [38], which is consistent with population surveys showing that first time women cannabis users were increasing at a faster rate than men $[10,13]$.

Our results also suggest the preferences for type and composition of cannabis used is shifting away from herbal cannabis to cannabis oil. In 2020, cannabis oil use declined compared with 2019 , which may be attributed to the increasing variety of cannabis delivery methods such as topical and edible forms that were not tracked in our sample. The composition of medical cannabis preferred by older adults was similar in a study by Brown and colleagues who found CBD-only products were used by $45.0 \%$ older adults while CBD:THC combinations were used by $21.7 \%$ [27]. The preference for CBD products was reported to be higher (57.0\%) in first time older adult users compared to those with past use (28.0\%) [36], though another study reported a preference $(82.0 \%)$ for THC-rich cannabis strains [26].

Use of medical cannabis by older adults may be partly driven by self-reported improvements in symptoms [26, 34, $35,38]$. In a previous study of 1186 older adults consulting a cannabis clinic, $59.1 \%$ considered the treatment successful, and $93.7 \%$ reported an improvement in their condition after 6 months [26]. Similar results were found in other studies-83\% $(n=345)$ [35], 80\% $(n=1000)$ [34], and 79\% $(n$ =274) [38] reported improvement in pain. Along with pain relief, older users also report stopping or reducing the dose of opioid medications (88\% [34], 18.1\% [26]). Across age groups, meta-analyses found moderate to low-quality evidence for pain relief with cannabis whereas the evidence for other symptoms was considered weak [29, 40]. Substitution of opioid mediations with cannabis was also reported across age groups [41-43].

Despite potential benefits, concerns remain regarding possible harms [29, 40]. A small but substantial proportion of users in our sample (7.9\%, Fig. 3) and in a previous study across all age groups [42] reported increasing opioid medications after using cannabis. Among older adults, adverse effects of medical cannabis such as drowsiness and dizziness $[35,36]$ are particularly concerning due to the risk of falls and impaired driving, as suggested by the higher rate of injuries and emergency department visits among older adults aged 50 years and above [44]. The improving risk perception associated with cannabis among older adults may lead to use of higher doses of cannabis and, consequently, to higher rates of these adverse effects [45]. Furthermore, stopping cannabis can be associated with poor sleep and anxiety [46], prompting continued usage and dependency. The 
resulting long-term use may have adverse effects that are not well recognized, though modest impairment in cognitive function with high doses of cannabis has been reported [47]. High-quality research is needed to determine safe cannabis use among older adults.

Limitations: This study used a convenience sample that may lead to over-representation of benefits due to more attrition of users with poor outcomes. Data collected in this sample were primarily for use by the medical cannabis clinic and its physicians, and adapted over time to meet the needs of the clinic, resulting in changes to the questions that may have affected user responses. Although changes to the questionnaires (provided in the electronic supplementary material, 'SI Methods') were largely consistent with previous versions, the influence of these changes on user responses cannot be ruled out. In addition, improvement in symptoms were assessed by self-report and only at follow-up; as symptoms were not assessed at baseline, an analysis of change in symptoms over time could not be performed. The data may also be geographically biased as respondents were predominantly from four provinces. However, our results were largely in line with previous studies from other countries as well as from community studies [26, 27, 35]. Finally, the exact doses of THC:CBD and strains of cannabis used could not be determined, and we could not rule out if users consumed additional cannabis products. As such, the dose of cannabis consumed could not be determined.

\section{Conclusion}

Cannabis use is increasing among older adults in Canada and worldwide [13, 48, 49]. Despite the tendency of older adults to source cannabis from a medical dispensary (50.3\%) [13], the widespread availability and social acceptance of cannabis may shift usage patterns [38]. Our data indicate that patterns of cannabis use are changing. Given that receiving advice from informal sources increases misinformation [50], the use of cannabis clinics by older adults represents an opportunity for improving safe cannabis use. However, challenges in obtaining medical advice persist [19], and highquality evidence is needed to support guidelines for clinical use $[32,51]$. While our results support the self-perceived effectiveness of medical cannabis, a small proportion of older adults report worsening symptoms, adverse effects, and required increased medication. Given these data, we support the call for high-quality evidence for medical cannabis use among older adults to enable informed evidencedbased clinical use.

Supplementary Information The online version contains supplementary material available at https://doi.org/10.1007/s40266-021-00913-y.

\section{Declarations}

Funding The authors report research support from the Alzheimer's Drug Discovery Foundation (grant no. GC-201808-2016354), Weston Foundation (grant no. CT190002), and the Canadian Consortium for Neurodegeneration in Aging (CCNA) to $\mathrm{NH}$ and KL, and through a CCNA fellowship to ST. The funders had no role in the design or conduct of the study, nor in the writing or approval for publication of the manuscript.

Competing interests $\mathrm{AD}$ consulted on unrelated projects with Canabo Medical, a subsidiary of Aleafia Health, a publicly traded company, which overlapped with the period of data collection. AD has no current financial relationship with either Aleafia Health or with Canabo Medical, and did not receive financial or other considerations for this article. The rest of the authors declare no competing interest.

Ethics approval The study was approved by the Sunnybrook Research Ethics Board.

Consent to participate All participants consented to participating in the data collection and sharing anonymized data for research purposes.

Consent for publication All authors approved the final version for publication.

Availability of data and material The data that support the findings of this study were obtained from Aleafia Health and are not publicly available. Further analyses of data reported in this study are available from the authors upon reasonable request and with permission of Aleafia Health.

Code availability STATA 15.1 was used to extract data with in-built commands, which can be made available on reasonable request to the authors.

Author contributions ST, KL, AD, and NH contributed to the conception and design of the work. AD analyzed the data, ST, KL, AD, and $\mathrm{NH}$ interpreted the data. The first draft of the manuscript was written by ST and RW. ST, KL, RW, AL, AD, and NH critically reviewed the manuscript.

\section{References}

1. Bridgeman MB, Abazia DT. Medicinal Cannabis: history, pharmacology, and implications for the acute care setting. Pharm Ther. 2017;42:180-8.

2. Levine HG. Global drug prohibition: its uses and crises. Int J Drug Policy. 2003;14:145-53. https://doi.org/10.1016/S0955-3959(03) 00003-3.

3. Fazey CSJ. The commission on narcotic drugs and the United Nations International Drug Control Programme: politics, policies and prospect for change. Int J Drug Policy. 2003;14:155-69. https://doi.org/10.1016/S0955-3959(03)00004-5.

4. Fischer B, Kuganesan S, Room R. Medical Marijuana programs: implications for cannabis control policy-observations from Canada. Int J Drug Policy. 2015;26:15-9. https://doi.org/10.1016/j. drugpo.2014.09.007.

5. Health Canada. Understanding the New Access to Cannabis for Medical Purposes Regulations [Internet]. aem. 2016. https://www.canada.ca/en/health-canada/services/publications/ 
drugs-health-products/understanding-new-access-to-cannabisfor-medical-purposes-regulations.html. Accessed 11 May 2021

6. Health Canada. Data on cannabis for medical purposes [Internet]. 2020. https://www.canada.ca/en/health-canada/services/ drugs-medication/cannabis/research-data/medical-purpose.html. Accessed 28 Jul 2021

7. Rotermann M, Langlois K. Prevalence and correlates of marijuana use in Canada, 2012. Health Rep. 2015;26:10-5.

8. Government of Canada. Cannabis Legalization and Regulation [Internet]. 2018. https://www.justice.gc.ca/eng/cj-jp/cannabis/. Accessed 11 May 2021

9. Hajizadeh M. Legalizing and regulating marijuana in Canada: review of potential economic, social, and health impacts. Int $\mathrm{J}$ Health Policy Manag. 2016;5:453-6. https://doi.org/10.15171/ ijhpm.2016.63.

10. Rotermann M. Looking back from 2020, how cannabis use and related behaviours changed in Canada. Health Rep. 2021;32:3-14. https://doi.org/10.25318/82-003-x202100400001-eng.

11. Han BH, Sherman S, Mauro PM, Martins SS, Rotenberg J, Palamar JJ. Demographic trends among older cannabis users in the United States, 2006-13. Addiction. 2017;112:516-25. https:// doi.org/10.1111/add.13670.

12. Salas-Wright CP, Vaughn MG, Cummings-Vaughn LA, Holzer KJ, Nelson EJ, AbiNader M, et al. Trends and correlates of marijuana use among late middle-aged and older adults in the United States, 2002-2014. Drug Alcohol Depend. 2017;171:97-106. https://doi. org/10.1016/j.drugalcdep.2016.11.031.

13. Statistics Canada. National Cannabis Survey, third quarter 2019 [Internet]. 2019. https://www150.statcan.gc.ca/n1/daily-quotidien/ 191030/dq191030a-eng.htm. Accessed 20 May 2021

14. Hazekamp A, Ware MA, Muller-Vahl KR, Abrams D, Grotenhermen $\mathrm{F}$. The medicinal use of cannabis and cannabinoids-an international cross-sectional survey on administration forms. J Psychoact Drugs. 2013;45:199-210. https://doi.org/10.1080/ 02791072.2013.805976.

15. Kaskie B, Ayyagari P, Milavetz G, Shane D, Arora K. The increasing use of cannabis among older Americans: a public health crisis or viable policy alternative? Gerontologist. 2017;57:1166-72. https://doi.org/10.1093/geront/gnw166.

16. Hasin DS, Saha TD, Kerridge BT, Goldstein RB, Chou SP, Zhang $\mathrm{H}$, et al. Prevalence of marijuana use disorders in the United States between 2001-2002 and 2012-2013. JAMA Psychiat. 2015;72:1235-42. https://doi.org/10.1001/jamapsychiatry.2015. 1858.

17. Health Canada. Canadian Cannabis Survey 2020: Summary [Internet]. 2020. https://www.canada.ca/en/health-canada/servi ces/drugs-medication/cannabis/research-data/canadian-canna bis-survey-2020-summary.html. Accessed 20 May 2021

18. Treister-Goltzman Y, Freud T, Press Y, Peleg R. Trends in publications on medical cannabis from the year 2000. Popul Health Manag. 2019;22:362-8. https://doi.org/10.1089/pop.2018.0113.

19. Baumbusch J, Yip IS. Exploring new use of cannabis among older adults. Clin Gerontol. 2021;44:25-31. https://doi.org/10.1080/ 07317115.2020.1746720.

20. Bobitt J, Qualls SH, Schuchman M, Wickersham R, Lum HD, Arora K, et al. Qualitative analysis of cannabis use among older adults in Colorado. Drugs Aging. 2019;36:655-66. https://doi.org/ 10.1007/s40266-019-00665-w.

21. Manning L, Bouchard L. Medical cannabis use: exploring the perceptions and experiences of older adults with chronic conditions. Clin Gerontol. 2021;44:32-41. https://doi.org/10.1080/07317115. 2020.1853299

22. Ng JY, Gilotra K, Usman S, Chang Y, Busse JW. Attitudes toward medical cannabis among family physicians practising in Ontario, Canada: a qualitative research study. CMAJ Open. 2021;9:E3428. https://doi.org/10.9778/cmajo.20200187.
23. Allan GM, Ramji J, Perry D, Ton J, Beahm NP, Crisp N, et al. Simplified guideline for prescribing medical cannabinoids in primary care. Can Fam Physician. 2018;64:111-20.

24. Haug NA, Padula CB, Sottile JE, Vandrey R, Heinz AJ, BonnMiller MO. Cannabis use patterns and motives: a comparison of younger, middle-aged, and older medical cannabis dispensary patients. Addict Behav. 2017;72:14-20. https://doi.org/10.1016/j. addbeh.2017.03.006.

25. Choi NG, DiNitto DM. Comparing older nonmedical and medical cannabis users: health-related characteristics, cannabis use patterns, and cannabis sources. Am J Drug Alcohol Abuse. 2021;47:612-22. https://doi.org/10.1080/00952990.2021.19083 18.

26. Abuhasira R, Schleider LB-L, Mechoulam R, Novack V. Epidemiological characteristics, safety and efficacy of medical cannabis in the elderly. Eur J Intern Med. 2018;49:44-50. https://doi.org/ 10.1016/j.ejim.2018.01.019.

27. Brown JD, Costales B, van Boemmel-Wegmann S, Goodin AJ, Segal R, Winterstein AG. Characteristics of older adults who were early adopters of medical cannabis in the Florida medical marijuana use registry. J Clin Med. 2020;9:1166. https://doi.org/10. 3390/jcm9041166.

28. Velayudhan L, McGoohan K, Bhattacharyya S. Safety and tolerability of natural and synthetic cannabinoids in adults aged over 50 years: a systematic review and meta-analysis. PLoS Med. 2021;18: e1003524. https://doi.org/10.1371/journal.pmed.10035 24.

29. Whiting PF, Wolff RF, Deshpande S, Di Nisio M, Duffy S, Hernandez AV, et al. Cannabinoids for medical use: a systematic review and meta-analysis. JAMA. 2015;313:2456-73. https:// doi.org/10.1001/jama.2015.6358.

30. Briscoe J, Casarett D. Medical marijuana use in older adults. J Am Geriatr Soc. 2018;66:859-63. https://doi.org/10.1111/jgs.15346.

31. Beauchet O. Medical cannabis use in older patients: update on medical knowledge. Maturitas. 2018;118:56-9. https://doi.org/10. 1016/j.maturitas.2018.10.010.

32. Fitzcharles M-A, Shir Y, Häuser W. Medical cannabis: strengthening evidence in the face of hype and public pressure. CMAJ. 2019;191:E907-8. https://doi.org/10.1503/cmaj.190509.

33. National Survey of Drug Use and Health. 2019 NSDUH Annual National Report I CBHSQ Data [Internet]. https://www.samhsa. gov/data/report/2019-nsduh-annual-national-report. Accessd 9 Nov 2021

34. Bachhuber M, Arnsten JH, Wurm G. Use of cannabis to relieve pain and promote sleep by customers at an adult use dispensary. $\mathrm{J}$ Psychoact Drugs. 2019;51:400-4. https://doi.org/10.1080/02791 072.2019.1626953.

35. Reynolds IR, Fixen DR, Parnes BL, Lum HD, Shanbhag P, Church $\mathrm{S}$, et al. Characteristics and patterns of marijuana use in community-dwelling older adults. J Am Geriatr Soc. 2018;66:2167-71. https://doi.org/10.1111/jgs.15507.

36. Yang KH, Kaufmann CN, Nafsu R, Lifset ET, Nguyen K, Sexton $\mathrm{M}$, et al. Cannabis: an emerging treatment for common symptoms in older adults. J Am Geriatr Soc. 2021;69:91-7. https://doi.org/ 10.1111/jgs.16833.

37. Sarvet AL, Wall MM, Keyes KM, Olfson M, Cerdá M, Hasin DS. Self-medication of mood and anxiety disorders with marijuana: higher in states with medical marijuana laws. Drug Alcohol Depend. 2018;186:10-5. https://doi.org/10.1016/j.drugalcdep. 2018.01.009.

38. Lum HD, Arora K, Croker JA, Qualls SH, Schuchman M, Bobitt J, et al. Patterns of marijuana use and health impact: a survey among older Coloradans. Gerontol Geriatr Med. 2019;5:2333721419843707. https://doi.org/10.1177/2333721419 843707. 
39. Hamilton HA, Brands B, Ialomiteanu AR, Mann RE. Therapeutic use of cannabis: prevalence and characteristics among adults in Ontario, Canada. Can J Public Health. 2017;108:e282-7. https:// doi.org/10.17269/cjph.108.6130.

40. Mücke M, Phillips T, Radbruch L, Petzke F, Häuser W. Cannabisbased medicines for chronic neuropathic pain in adults. Cochrane Database Syst Rev. 2018;3(3): CD012182. https://doi.org/10. 1002/14651858.cd012182.pub2.

41. Corroon JM, Mischley LK, Sexton M. Cannabis as a substitute for prescription drugs-a cross-sectional study. J Pain Res. 2017;10:989-98. https://doi.org/10.2147/jpr.s134330.

42. Ishida JH, Wong PO, Cohen BE, Vali M, Steigerwald S, Keyhani S. Substitution of marijuana for opioids in a national survey of US adults. PLoS ONE. 2019;14: e0222577. https://doi.org/10.1371/ journal.pone.0222577.

43. Sexton M, Cuttler C, Finnell JS, Mischley LK. A cross-sectional survey of medical cannabis users: patterns of use and perceived efficacy. Cannabis Cannabinoid Res. 2016;1:131-8. https://doi. org/10.1089/can.2016.0007.

44. Choi NG, Marti CN, DiNitto DM, Choi BY. Older adults' marijuana use, injuries, and emergency department visits. Am J Drug Alcohol Abuse. 2018;44:215-23. https://doi.org/10.1080/00952 990.2017.1318891.

45. Han BH, Funk-White M, Ko R, Al-Rousan T, Palamar JJ. Decreasing perceived risk associated with regular cannabis use among older adults in the United States from 2015 to 2019. J Am Geriatr Soc. 2021;69(9):2591-7. https://doi.org/10.1111/jgs.17213.

46. Perron BE, Holt KR, Yeagley E, Ilgen M. Mental health functioning and severity of cannabis withdrawal among medical cannabis users with chronic pain. Drug Alcohol Depend. 2019;194:401-9. https://doi.org/10.1016/j.drugalcdep.2018.09.029.

47. Scott EP, Brennan E, Benitez A. a systematic review of the neurocognitive effects of cannabis use in older adults. Curr Addict Rep. 2019;6:443-55. https://doi.org/10.1007/s40429-019-00285-9.

48. Lloyd SL, Striley CW. Marijuana use among adults 50 years or older in the 21 st century. Gerontol Geriatr Med. 2018;4:2333721418781668. https://doi.org/10.1177/2333721418 781668.

49. Lowry DE, Corsi DJ. Trends and correlates of cannabis use in Canada: a repeated cross-sectional analysis of national surveys from 2004 to 2017. CMAJ Open. 2020;8:E487-95. https://doi. org/10.9778/cmajo.20190229.

50. Ishida JH, Zhang AJ, Steigerwald S, Cohen BE, Vali M, Keyhani S. Sources of information and beliefs about the health effects of marijuana. J Gen Intern Med. 2020;35:153-9. https://doi.org/10. 1007/s11606-019-05335-6.

51. Bertram JR, Porath A, Seitz D, Kalant H, Krishnamoorthy A, Nickerson J, et al. Canadian guidelines on cannabis use disorder among older adults. Can Geriatr J. 2020;23:135-42. https://doi. org/10.5770/cgj.23.424. 